\title{
Inverse Magnetorheological Fluids
}

\section{Rodríguez-Arco, ${ }^{a}$ M.T. López-López, ${ }^{* a}$ A.Y. Zubarev,${ }^{b}$ K. Gdula ${ }^{c}$ and J.D.G. Durán ${ }^{a}$}

We report a new kind of field-responsive fluids consisting of suspensions of diamagnetic (DM) and ferromagnetic (FM) microparticles in ferrofluids. We designate them as inverse magnetorheological (IMR) fluids for analogy with inverse ferrofluids (IFFs). Observations on the particle self-assembly in IMR fluids upon magnetic field application showed that DM and FM microparticles were assembled into alternating chains oriented along the field direction. We explain such assembly on the basis of the dipolar interaction energy between particles. We also present results on the rheological properties of IMR fluids and, for comparison, of IFFs and bidispersed magnetorheological (MR) fluids. Interestingly, we found that upon magnetic field, the rheological properties of IMR fluids were enhanced with respect to bidispersed MR fluids with the same FM particle concentration, by an amount greater than the sum of the isolated contribution of DM particles. Furthermore, the fieldinduced yield stress was moderately increased when up to $30 \%$ of the total FM particle content was replaced with DM particles. Beyond this point, the dependence of the yield stress on the DM content was non-monotonic, as expected for FM concentrations decreasing to zero. We explain these synergistic results by two separate phenomena: the formation of exclusion areas for FM particles due to the perturbation of the magnetic field by DM particles, and the dipole-dipole interaction between DM and FM particles, which enhances the field-induced structures. Based on this second phenomenon, we present a theoretical model for the yield stress that semi-quantitatively predicts the experimental results.

\section{Introduction}

Controlling the interactions among particles in colloidal systems has attracted much attention in recent years. ${ }^{1-9}$ Such an interest is mainly due to the possibility of using these systems as smart materials with user-tuneable properties. In addition, it makes possible the bottom-up fabrication of materials with organised structures or patterns. This is achieved by controlling the positions, orientations and assembly of the particles. ${ }^{1-9}$

One of the most advantageous tools to externally self-assemble particles is the use of electric or magnetic fields. An important benefit related to them is their reversibility, which allows rapidly returning to the disordered state when the field is switched off. Other advantages are (i) compatibility for wet and dry environments, (ii) lack of necessity of physical contact for assembly and, (iii) easiness to scale from nano-/meso- to macroscale. ${ }^{3,4}$ The basis of this type of assembly is the dipole-dipole interaction among the particles. When polarisable/magnetisable particles are dispersed in a continuous medium under an external field, they acquire an electric/magnetic moment which makes them interact. As a result, they form particle chains that can evolve to much more complex structures such as percolated chains. The dipole-dipole interaction can be easily modified by changing the shape, composition, dimensions and electric permittivity/magnetic permeability of the dispersed particles or the electric/magnetic properties of the continuous medium. Therefore there is a wide range of possibilities to control particle interactions and many examples of fieldresponsive fluids and field-induced self-assembly have been reported in the literature. ${ }^{3-11}$

A particularly powerful system for the magnetic assembly of colloidal microparticles is ferrofluids (FFs). FFs are suspensions of nanometric magnets in a liquid carrier and, as a result, they behave as continuous hydrodynamic and magnetic media for micron-sized particles dispersed in them. The simplest systems are the dispersions of either ferromagnetic (FM) or diamagnetic (DM) microparticles in FFs. In the first case we have the so-called extremely bimodal (or bidispersed) magnetorheological (MR) fluids. ${ }^{12-14}$ These kinds of MR 
fluids present some interesting structural properties, such as the fact that at close distances, FM microparticles dispersed in the FF can repel each other instead of being attracted upon the application of a magnetic field. Such a phenomenon is controlled by the formation of clouds by the FF nanoparticles around the FM microparticles. ${ }^{10}$ Furthermore, when DM microparticles are dispersed in a FF, a complex fluid known as inverse ferrofluid (IFF) is obtained. The main interest of IFFs is as model systems for MR fluids, because DM particles are usually smoother and more monodispersed than FM particles, and they also develop an effective magnetic moment when dispersed in a magnetic continuum. ${ }^{15,16}$ More complex systems using FFs as carriers are obtained when two populations of microparticles characterised by a different magnetic nature are dispersed jointly. For example, Erb et al. dispersed DM and paramagnetic particles in a magnetised FF. By controlling the magnetic permeability of both the FF and the dispersed particles, they obtained self-assembled structures such as Saturn rings, flower-like structures and multipole arrangements among others. ${ }^{7}$ Similarly, Khalil et al. also used paramagnetic and DM particles to assemble over twenty pre-programmed structures in a FF. ${ }^{8}$ Note that for these systems, theoretical simulations by Liu et al. predicted an enhancement of the magneto-induced stress. ${ }^{17}$ In addition, cell manipulation has profited from FFs by means of negative magnetophoresis, in which the environment (i.e., the FF) has higher magnetic permeability than the cells. For example, Krebs et al. reported the formation of linear chains of cells in a FF. These chains were adhered to cell-adhesive surfaces and kept on growing even after removing the FF and the magnetic field. ${ }^{18}$

The present work is focused on a new type of field-responsive fluid, consisting of FM and DM particles dispersed jointly in a FF. For analogy with IFFs, from now on we will refer to these suspensions as inverse magnetorheological (IMR) fluids. In this work, we first analyse the internal microstructure (particle assembly) of these new IMR fluids upon magnetic field application. We explain the resulting structures on the basis of the dipole-dipole interaction energy among the dispersed particles. In addition, we study the particle assembly effects on some technological-related properties of the suspensions, namely their rheological -flowproperties. More specifically, we show that the field-induced rigidity and the yield stress of IMR fluids are considerably enhanced with respect to suspensions of FM microparticles in FFs (i.e., extremely bimodal MR fluids). To the best of our knowledge, IMR fluids have never been studied before in the literature, in spite of their promising technological applications.

\section{Experimental}

We prepared IMR fluids by dispersing DM and FM microparticles in a FF. For comparison, we also prepared IFFs and extremely bimodal MR fluids by the dispersion of, respectively, DM and FM microparticles in a FF. As microparticles we chose PMMA (Microbeads, Spheromers CA10) and iron (BASF, HS quality) powders of diameters $9.9 \pm 0.4 \mu \mathrm{m}$ and $1.0 \pm 0.7 \mu \mathrm{m}$ respectively. We labelled all the suspensions in the general form FMX-DMY, where $\mathrm{X}$ and $\mathrm{Y}$ stood for the FM and DM particle volume fractions respectively. The FF was prepared as described in ref.19 and consisted of 5 vol \% of oleate-coated magnetite nanospheres -particle diameter of $8.7 \pm 2.0 \mathrm{~nm}$ - dispersed in mineral oil (Sigma Aldrich).

We conducted microscopic observations of the samples in the absence of external magnetic fields and upon their application. For this purpose, we placed a Haake MARS III Rheoscope Module (Thermo Fisher Scientific, USA) between two Helmholtz coils whose main axis was perpendicular to the axis of the microscope. We measured the rheological properties of all the samples using a Haake Mars III rheometer (Thermo Fisher Scientific, 
USA). The gap between the two rough plates of the measuring system was $400 \mu \mathrm{m}$. The major source of error when using this type of geometry is connected to the operator error associated to the filling of the sample. In order to quantify the importance of such an error we performed several measurements filling the gap with different freshly prepared samples. The so-estimated error for the measured rheological quantities is taken into account in the experimental uncertainties shown in the figures of this manuscript.

We conducted the rheological characterization upon magnetic field application by using a coil that applied a homogeneous magnetic field perpendicular to the plates of the rheometer. We firstly performed two types of steady-state measurements (controlled shear rate or controlled shear stress) using the protocol that follows: (i) pre-shear: $30 \mathrm{~s}$ of $150 \mathrm{~s}-1$ shear rate application; (ii) waiting time: we turned on the magnetic field and the sample was left at rest for 2 minutes; (iii) depending on the type of measurement, we subjected the sample to a linear ramp of shear rates (controlled shear rate measurements) or stresses (controlled shear stress measurements) with a duration of $30 \mathrm{~s}$ for each step, upon the same magnetic field as in (ii). The shear rate ramps (from 20 to $300 \mathrm{~s}-1$ ) were performed in order to determine, as it will be explained below, the dynamic yield stress of the suspensions, while the so-called static yield stress was obtained from shear stress ramps. Secondly, we carried out oscillatory measurements with the same pre-shear and waiting time as in the steady state measurements, followed by a logarithmic ramp of sinusoidal stresses of fixed frequency, $\mathrm{f}=1 \mathrm{~Hz}$, and increasing values of the stress amplitude, $\sigma 0$. The sinusoidal stress at each step was applied during 8 periods of oscillations. We recorded the resulting viscoelastic moduli - i.e., the storage, G', and loss, G', moduli-during the last 5 periods.

\section{Results and discussion}

The microscopic observations of the IMR fluids showed that in the presence of an external magnetic field, both populations of particles, FM and DM, built chain-like structures in the field direction. In the absence of field, the FM and DM particles inside sample FM10-DM20, for example, appeared randomly distributed (Fig. 1a). However, when the field was activated, the DM particles, of bigger size, formed single particle chains aligned with the field (Fig. 1b). The motion of the DM particles in order to build long, continuous, DM chains more perfectly aligned with the magnetic field appeared to be hindered, contrary to what happened in the case of the IFF, FM0-DM10 (Fig. 1c). The likely reason for such behaviour was the impediment offered by clusters of FM particles placed at both sides of the DM chains. Indeed, the FM particles, of a smaller size, built clusters of chains oriented in the field direction, rather than single particle chains. Such clusters were attracted to the laterals of the DM single chains (Fig. 1b and more clearly seen in Fig. 1d). As a result, the initially isotropic suspension became strongly patterned with the formation of alternating bands of FM and DM particles when the magnetic field was connected.

The FM particles in the FM clusters or bands appeared to be densely packed, presumably with a concentration close to the maximum packing fraction of spheres $(\sim 0.7)$, with almost no free space between the chains. Such a distribution was rather different to that found for a sample without DM particles and the same concentration of FM particles, FM10-DM0, despite using the same volume of sample for the observations. In this latter case, the FM chains were more separated and zones of pure FF frequently appeared between chains (Fig. 1e). For the IMR fluids, pure FF spaces without FM microparticles were almost only found (i) at the interstices between DM particles of the same chain -highlighted in the sketch of the particle structures of Fig. 1f- and (ii) at the ends of the DM chains -highlighted in the sketch 
of the particle structures of Fig. 1f. Therefore, in the IMR fluids, the FM chains packed together into dense zones and formed some sort of "caves" to house the DM chains.

We can give an explanation to the formation of the FM caves around the DM chains based on the simple dipole-dipole interaction among the dispersed particles. In the case of magnetic fields, the magnetic moment acquired by an isolated particle of relative magnetic permeability ${ }^{\mu}$ when placed in an external magnetic field $\mathrm{H} 0$, is $\mathbf{m}=3 \mu_{f} \beta_{p} V \mathbf{H}_{0}$. Here $\mathrm{V}, \mu_{f}$ and $\beta_{p}=\frac{\mu_{p}-\mu_{f}}{\mu_{p}+2 \mu_{f}}$ stand for the particle volume, the relative magnetic permeability of the carrier medium and the magnetic contrast factor, respectively.20 In the case of FFs, $\mu_{f}>1$, in comparison to non-magnetic liquids such as oil, water, etc. for which ${ }^{\mu_{f}}=1$. Actually, ${ }^{\mu_{f}}$ can be adjusted by varying the concentration of the FF which is interesting for self-assembly purposes as manifested in the work of Erb et al.7 In the case of DM particles dispersed in a $\mathrm{FF}, \beta_{D M}<0$, because for them

$\mu_{p}=1$. On the contrary, for the FM ones, $\beta_{F M} \rightarrow 1$, because in this case $\mu_{p}>>1$. As a result, the DM particles in the FF did acquire an effective magnetic moment that opposed to the external field. The FM particles also acquired a magnetic moment, but in their case, aligned with the field. Due to their magnetised state, dipole-dipole interaction among the dispersed particles took place. The interaction force on a particle $\delta$ due to the presence of a particle $\alpha$ of magnetic moments, $\mathbf{m}_{\delta}$ and $\mathbf{m}_{\alpha}$ respectively, separated by a vector $\mathbf{r}$ which connects the centre of particle $\alpha$ to the centre of particle $\delta$ can be estimated as:21

$\mathbf{F}_{a \tilde{\delta}}=\frac{\mu_{0}}{4 \pi \mu_{f}}\left\{3 \frac{\mathbf{m}_{a} \cdot \mathbf{m}_{\delta}}{r^{5}} \mathbf{r}+\frac{3}{r^{5}}\left[\left(\mathbf{m}_{a} \cdot \mathbf{r}\right) \mathbf{m}_{\hat{\delta}}+\left(\mathbf{m}_{\hat{\delta}} \cdot \mathbf{r}\right) \mathbf{m}_{a}\right]-\frac{15}{r^{7}}\left(\mathbf{m}_{\alpha} \cdot \mathbf{r}\right)\left(\mathbf{m}_{\hat{\delta}} \cdot \mathbf{r}\right) \mathbf{r}\right\}$

where $\mu_{0}$ stands for the magnetic permeability of vacuum. In order to analyse the assembly of the particles in the presence of a field, let us firstly consider the case of two particles belonging to the same population, that is, a pair of either two FM or two DM particles. In this case, their magnetic moments are equal and aligned with the external field, $\mathbf{m}_{\alpha}=\mathbf{m}_{\delta} \uparrow \mathbf{H}_{\mathbf{0}}$. As a result, the net force on particle $\delta$ due to particle $\alpha$ is attractive, that is, opposing to $\mathrm{r}$, when the line-of-centres is parallel to the external field, while it is repulsive when it is perpendicular, as shown in Fig. 2. At an intermediate angle between these two extreme situations, the pair of particles is subjected to a magnetic torque which tends to align the line-of-centres with the field. Because of this reason, particles of the same population -either FM or DM particlesaligned and assembled into chain-like structures in the field direction.

If we consider now the interaction between two particles of different populations, that is, a pair of a FM particle and a DM particle, the interaction force is repulsive when the line-ofcentres is parallel to the magnetic field, while it becomes attractive when it is perpendicular Fig. 2. This is due to the opposite sense of their dipoles $\mathbf{m}_{\alpha} \uparrow \mathbf{m}_{\delta} \downarrow$. As a result, the DM particles placed next to the FM ones, side to side, with the centre-to-centre line perpendicular to the field, giving rise to the appearance of alternating bands of FM and DM chains. The fact that particles of different populations could not align in the same chain -i.e., the line-ofcentres being parallel to the field- also explained the appearance of those zones without FM microparticles at the ends of the DM chains seen above. Therefore, the simple dipole-dipole consideration explained the formation of the cave-like structures in IMR fluids.

Since the microscopic structure of a material determines its macroscopic rheological behaviour, we should expect differences among the samples consisting just of FM or DM particles and the IMR fluids. To corroborate this hypothesis, we first of all measured the 
steady-state rheological behaviour of the samples in the absence of a magnetic field and upon its application. In the absence of field, all the suspensions showed a Newtonian behaviour i.e., a linear relationship between the shear stress, $\sigma$, and the shear rate, $\dot{\gamma}$, with negligible intercept (Fig. 3). However, when the field was turned on the rheological behaviour changed to a plastic behaviour. The plastic behaviour is characterised by the appearance of a yield stress, $\sigma y$, that it is necessary to overcome to induce the flow -i.e., manifested by the intermediate pseudoplateau of the plots of Fig. 3. Such field-induced rigidity and resistance to the flow appear as a result of the microscopic structures formed by the dispersed particles in the field direction -i.e., perpendicular to the direction of the shear. The field-induced structures are reinforced when the magnetic field is increased, which explains the increase of the shear stress with the field. At a first glance to the rheograms $-\sigma$ vs. $\dot{\gamma}$ plots - of Fig. 3 , it seemed that such an increase was more important for the IMR fluids. For example, the shear stress of sample FM10-DM10 for given H0 and $\dot{\gamma}$ appeared to be generally higher than for sample FM10-DM0 (Fig. 3). Nevertheless, the best method to evaluate the strength of the particle structures is the estimation of the so-called yield stress.

Depending on the degree of rupture of the structures, we may define two yield stresses: (i) The static yield stress, related to the breakage of the structures at their weakest point, and obtained by extrapolating the shear stress at the intermediate pseudoplateau -i.e., $\dot{\gamma} \approx 0.1 \mathrm{~s}^{-1}-$ in log-log rheograms.22 (ii) The dynamic yield stress, related to the complete breakage of the structures, and usually estimated by fitting the high-shear rate part of the rheograms in linear scale $\left(\dot{\gamma}>200 \mathrm{~s}^{-1}\right)$ to Bingham equation, ${ }^{\sigma}=\sigma_{y}+\eta \dot{\gamma}$ where $\eta$ is the so-called plastic viscosity.22 We estimated both yield stresses and plotted them against the magnetic field strength for the samples of fix concentration of iron, $\varphi_{F M}=0.1$, and for sample FM0-DM10 in Fig. 4a.

Both the dynamic and the static yield stresses were higher for the IMR fluids and increased with the DM concentration, for a constant FM volume fraction and for all the magnetic fields (Fig. 4a). With respect to the dependence on the magnetic field, both the static and the dynamic yield stresses increased with the intensity of the field for all the samples (Fig. 4a). Such an increase is usually known as the magnetorheological (MR) effect and has important technological-related applications.20 The MR effect of sample FM10-DM20 was the highest one, followed by sample FM10-DM10. The yield stresses of samples FM10-DM0 and FM0DM10 were significantly lower. Actually, for sample FM0-DM10 it was almost negligible for all the applied fields (Fig. 4a). The extremely low value of the yield stress in IFFs (i.e., like sample FM0-DM10) has been widely studied in the literature.15-16 Actually, it has been attributed to the very weak interactions between DM particles, in contrast to the strong magnetic interactions between particles of high magnetic permeability like the FM particles. The smallness of the yield stress of sample FM0-DM10 made evident that the increase of the yield stress of sample FM10-DM10 with respect to sample FM10-DM0 was not just the result of the addition of the yield stress of the chains of DM particles. In other words, the yield stress of sample FM10-DM10 at a particular field was not the sum of the yield stresses of samples FM10-DM0 and FM0-DM10 at the same field. Similarly, the increase for sample FM10-DM20 with respect to FM10-DM10 could be neither due just to the contribution of the DM chains. Consequently, the DM particles played a negligible role in the yield stress of IFF FM0-DM10, but, on the contrary, their role was very significant in IMR fluids. Therefore, there was a synergistic effect when mixing both particle populations.

However, a critical concentration of DM particles at which such a synergistic effect was maximal appeared when the total concentration of solids was fixed and the relative volume fractions of FM and DM particles were varied. For example, for a total volume fraction of 30 
$\%$ and at a given intensity of the magnetic field, the dynamic yield stress increased with the DM volume fraction until the latter reached approx. 10 vol \% (see Fig. 4b). This is a surprising result because, for example, sample FM20-DM10 exhibited higher yield stress than sample FM30-DM0, in spite of the fact that the concentration of FM particles was decreased by $33 \%$. Although not huge, such an increase is definitely significant enough (32\% at a magnetic field of $17.1 \mathrm{kA} / \mathrm{m}$ ), especially if we take into account that the replacement of magnetic particles with non-magnetic particles gives rise to an increase of a magnetic-related quantity. For increasing concentrations of DM particles, the dynamic yield stress diminished, as a result of the further reduction of the FM volume fraction. The effect of the $\varphi_{F M}$ reduction can be taken into account if we calculate a dimensionless dynamic yield stress defined as follows:

$s=\frac{8 \sigma_{y}^{d}}{9 \mu_{0} \mu_{f} \beta_{F M}{ }^{2} H_{0}^{2} \varphi_{F M}}$

A similar form of the dimensionless yield stress has been proposed in the literature for the static yield stress of suspensions of magnetic particles.20 In our case, the so-obtained experimental yield stress increased with the ratio of the DM volume fraction to the FM one, $x=\varphi_{D M} / \varphi_{F M}$ (also dimensionless), for constant $\varphi_{D M}+\varphi_{F M}=0.3$ (Fig. 5). The experimental data followed a single master curve for the different values of the external field, despite some dispersion due to slight deviations from the quadratic tendency of the yield stress on $\mathrm{H} 0$. Nevertheless the experimental increase of the dimensionless yield stress with increasing $\varphi_{D M}$ was clearly observed.

We also observed an increase of the dynamic (oscillatory) properties of the IMR fluids, in agreement with the steady-state results. In the absence of magnetic field, all the samples exhibited a liquid-like behaviour, characterised by higher values of $G$ ' ' with reference to $G$ ' (Fig. 6). We must take in mind that $G^{\prime}$ and $G$ ' ' respectively represent the elastic -i.e., solidlike- and the viscous -i.e., liquid-like-contributions to the stress response. 23 Therefore, this result agreed well with the above-described Newtonian behaviour of the samples at zero field. When the magnetic field was activated, both moduli increased for all the samples. For the samples with FM particles, $G$ ' became higher than $G$ ' ' until a critical value of the stress amplitude at which they crossed (Fig. 6). This meant that the samples with FM particles showed a solid-like behaviour until such a critical value, and a liquid-like one above it. Such a magnetic field-induced change of the oscillatory rheological behaviour was somewhat connected to the MR effect mentioned above. On the contrary, for sample FM0-DM10, G', remained higher than $G^{\prime}$ for all the magnetic fields (data not shown here for brevity), and therefore this sample preserved its liquid-like behaviour even when the field was applied. Again, such behaviour was connected to the almost negligible values of the yield stress found for sample FM0-DM10 in the steady-state measurements.

As it happened for the steady-state results there was always an enhancement of the suspension rigidity upon field application when FM and DM particles were mixed together. Such rigidity, usually quantified by $G^{\prime}$, is also related to the field-induced structures. Let us focus, first of all, on the situation in which the field was inactive. In this case, G' remained almost linear and thus, independent of the stress amplitude, for low values of $\sigma 0$ (Fig. 6). At higher values of $\sigma 0, G^{\prime}$ abruptly decreased and reached a local minimum. An explanation to such a minimum is currently lacking in the literature for suspensions of magnetic particles.24 After the minimum, $G$ ' slightly increased for the largest values of $\sigma 0$. When the magnetic field was activated, an additional quasi-plateau at intermediate values of the stress amplitude appeared only for the suspensions containing FM particles (Fig. 6). 
The appearance of such a second plateau has been previously reported in the literature for suspensions of FM particles. The initial decrease of $G^{\prime}$ after the first plateau has been attributed to short-scale rearrangements of the particles inside the field-induced structures.24,25 The second abrupt drop has been explained by large-scale rearrangements and rupture-reformation of the particle structures due to their homogeneous deformation.24,25 Therefore, the values of $G^{\prime}$ in the first plateau are an indication of the strength of the particle structures. As it happened for the yield stress, these values were higher for the IMR fluids and increased with the DM volume fraction in the whole range of magnetic fields. Likewise, the values of $G^{\prime}$ were negligible for sample FM0DM10 in comparison (Fig. 7). Again, the addition of DM particles strongly enhanced a magnetic field-related quantity, by an amount greater than the isolated contribution of the DM particles.

In addition to an increase of the rigidity upon field, the mixture of DM and FM particles gave rise to an increased dissipation of energy upon the oscillatory stimulus. The quantity related to such a dissipation of energy is the loss modulus, G', In the particular case of suspensions of FM particles, energy is essentially dissipated by two mechanisms: (i) viscous effects in the carrier liquid and (ii) contact friction between particles inside the fieldinduced aggregates. In suspensions of FM particles the latter usually dominates.26,27 In our case, G' at the initial plateau was higher for the samples with FM and DM particles and increased with the DM volume fraction. For sample FM10-DM0 was lower and for sample FM0-DM10 it was extremely low (Fig. 7). These results suggest that friction between FM particles inside the aggregates must have been higher for the IMR fluids -i.e., inside the above-seen cave-like structures.

As evidenced from all the experimental results, there is an enhancement of the MR properties of the suspensions when FM and DM particles are dispersed jointly. Such an enhancement goes beyond that corresponding to the sum of the MR effect of the DM particle chains, because for IFFs, these properties were extremely low. Two main reasons are likely responsible for this synergistic effect when DM particles are added to an extremely bimodal suspension. Firstly, the presence of DM particles gives rise to a perturbation of the magnetic field, which creates some exclusion areas where the formation of chains by the FM particles is hindered as evidenced in Fig. 1 and discussed in the related text. These exclusion areas imply an increased concentration of FM particles in the permitted areas (something observed in Fig. 1 too). Because the MR properties of MR suspensions increase faster than linear with the FM particle concentration,20 this could explain the enhancement observed in our case. The second mechanism would be the magnetic interaction among FM and DM particles, due to a strengthening of the resulting FM/DM structures.

\section{Theoretical model}

In this paragraph we analyse the extent of the influence of the magnetic interaction among the FM and DM particles on the improvement of the field-induced yield stress of the IMR fluids. For this purpose we develop a theoretical model just based on the formation of structures due to the interaction among the different types of particles. The theoretical consideration of the exclusion areas mentioned above would imply much more complex mathematics. Nevertheless, this simple assumption allows us to semi-quantitatively explain the enhancement of the yield stress with the DM particle concentration, and to estimate the influence of the exclusion areas for the FM particles, as we will see.

\subsection{Internal structures in the IMR fluids}


We consider here a system of micron-sized FM and DM particles suspended in a FF subjected to an external magnetic field, H0. The external magnetic field induces a magnetic moment mFM on the FM particles and an effective moment $\mathrm{mDM}$ on the DM particles (magnetic holes). For simplification, we consider that all microparticles have identical size and that they are big enough to neglect any Brownian effects in the system. We suppose that particles of the same population, FM or DM, form linear chains -i.e., similar to those shown in Fig. 1. Because the moments mFM and mDM have opposite directions, the FM and DM chains experience lateral attraction and aggregation as mentioned above.

The determination of the DM particle distribution on the FM chains is a rather difficult problem. However, it is clear that such a distribution depends on the ratio of the FM to the $\mathrm{DM}$ volume concentrations, $x=\varphi_{D M} / \varphi_{F M}$. For this reason, let us suppose that the inequality $\varphi_{F M}>\varphi_{D M}$ is held. We denote the numbers of FM and DM particles in the aggregate as NFM and NDM respectively. In the framework of the used approximations the following equality $N_{D M} / N_{F M}=\varphi_{D M} / \varphi_{F M}$ is held. In order to avoid very cumbersome mathematics, we suppose that each chain of FM particles aggregates with a single chain of DM particles. The hypotheses of a higher FM volume fraction and identical particle size for both particle populations lead to shorter DM chains in comparison to FM chains. Calculations of the magnetic interaction energy between the FM and NM chains show that the magnetic attraction between the FM and DM particles is maximal near the extremities of the chains. For this reason, we suppose that the extreme particles of the chains are in contact. As for the relative position of the chains, it is identical, from the energetic viewpoint that the DM chains place either near the bottom or near the upper point of the FM chains. From now on we suppose that the DM chains place near the upper point of the FM chains as shown in Fig. 8a, where we plot the supposed structure of the composite aggregate and its deviation from the direction of the applied field HO under the action of the macroscopic shear flow.

To simplify calculations, we will use here approximations similar to those employed in the well-known theory for the magnetoviscous effect in electro- and magnetorheological fluids.28 For this reason, we first suppose that the magnetic moments of all the particles in the chains have the same magnitude and direction as those of the isolated single particles. Secondly, we only take into account magnetic interactions among the nearest particles in the aggregate.

When the aggregate deviates from the applied field by an angle $\theta$ (Fig. 8a), the angle $\alpha$ between the aggregate axis and the line connecting the centres of the nearest FM and DM, changes from its equilibrium value in the absence of shear $(\pi / 2)$. Nevertheless, simple calculations demonstrate that the change of $\alpha$ with $\theta$ is negligible. The macroscopic magnetoviscous effect is thus determined by the size of the aggregate and the angle $\theta$. This latter can be found from the balance between the magnetic and hydrodynamic torques acting on the aggregate. The magnetic torque, $\Gamma_{m}$, is estimated from the energy of magnetic interaction among the particles in the cluster, $\mathrm{U}$, which can be calculated using the simple dipole-dipole approximation:28

$\Gamma_{m}=-\frac{\partial U}{\partial \theta}=-6 \lambda\left[N_{F M}-1+y^{2}\left(N_{D M}-1\right)+y N_{D M}\right] \sin \theta \cos \theta$

with $\lambda=\frac{\mu_{0}}{4 \pi \mu_{f}} \frac{m_{F M}^{2}}{d^{3}}$ and $y=\frac{m_{D M}}{m_{F M}}$, $\mathrm{d}$ being the particle diameter, mFM and mDM the absolute magnitudes of the FM and DM particle moments respectively. In the Cartesian coordinate system (Fig. 8a), the velocity of the shear flowing suspension can be presented as $\mathrm{v}_{\mathrm{x}}=\dot{\gamma} z$. Let 
the velocity of the aggregate motion be v0. The hydrodynamic torque ${ }^{\Gamma_{h}}$, which compensates the magnetic torque, must be determined with respect to the particle of the cluster with the coordinate $z_{0}$ satisfying the condition $\dot{\gamma} z_{0}=\mathrm{v}_{0}$. This point ("particle of equal forces", designated as $\mathrm{kc}$ ) is determined from the condition that the total hydrodynamic drag force, acting on the particles above kc equals to the force, acting on the particles below $\mathrm{kc}$. Depending on the ratio of NDM to NFM, the particle with number kc can lie either below the chain of the DM particles $(\mathrm{kc}=\mathrm{k} 1$ in Fig. $8 \mathrm{~b})$, or inside the region of the "double" chain (kc $=\mathrm{k} 2$ ). We shall consider these two situations separately.

a. The particle of equal forces lies "below" the DM particle chain and $\mathrm{kc}=\mathrm{k} 1$. The hydrodynamic force, approximated as the classical Stokes force, can be estimated as in ref.28. Upon this approximation, we obtain the forces $F_{1 x}^{\prime}$ and $F_{1 x}^{\prime \prime}$, for particles with numbers from 1 to $\mathrm{k} 1$ and for particles situated above $\mathrm{k} 1$, respectively:

$F_{1 x}^{\prime}=\beta d \dot{\gamma} \cos \theta \sum_{i=1}^{k_{1}} i \quad F_{1 x}^{\prime \prime}=\beta d \dot{\gamma} \cos \theta\left(\sum_{i=1}^{N_{F M}-k_{1}} i+\sum_{i=N_{F M}-N_{D M}-k_{1}}^{N_{F M}-k_{1}} i\right)$

With $\beta=3 \pi \eta_{0} d, \eta_{0}$ being the viscosity of the carrier liquid. Equating $F_{1 x}^{\prime}$ to $F_{1 x}^{\prime \prime}$, we obtain an equation on $\mathrm{k} 1$. Considering that strong magnetoviscous effects can only be provided by long enough chains, i.e. ${ }^{N_{D M}}, N_{F M}>>1$, we finally get:

$k_{1} \approx \frac{N_{F M}}{2} \frac{1+2 x-x^{2}}{1+x}$

Where $x=N_{D M} / N_{F M}=\varphi_{D M} / \varphi_{F M}$. We have supposed that the particle corresponding to $\mathrm{k} 1$, lies below the DM chain in Fig. 8. In other words, the inequality $k_{1}<N_{F M}-N_{D M}$ is fulfilled. By using (5), we find the criterion of this inequality: $x<\sqrt{2}-1$.

b. The particle of equal forces lies "inside" the region of the double chain $(\mathrm{kc}=\mathrm{k} 2)$ and $\mathbf{1}>\mathbf{x}>\sqrt{\mathbf{2}}-\mathbf{1}$. In this second case, instead of (4), we get respectively:

$F_{2 x}{ }^{\prime}=\beta \dot{\gamma} d \cos \theta\left(\sum_{i=1}^{k_{2}} i+\sum_{i=1}^{k_{2}-N_{\text {EM }}-N_{D M}} i\right) F_{2 x}{ }^{\prime \prime}=\beta \dot{\gamma} d \cos \theta 2 \sum_{i=1}^{N_{F M}-k_{2}} i$

Equating $F_{2 x}{ }^{\prime}$ to $F_{2 x}{ }^{\prime \prime}$ and taking into account the strong inequalities $N_{D M}, N_{F M}>>1$, we get:

$k_{2}=\frac{2 N_{F M}^{2}-\left(N_{F M}-N_{D M}\right)^{2}}{4 N_{F M}}$

The hydrodynamic torques $\Gamma_{h 1}$ and $\Gamma_{h 2}$, corresponding to the inequalities $x<\sqrt{2}-1$ and $1>x>\sqrt{2}-1$ respectively, can be estimated as: 28

$\Gamma_{h 1} \approx \beta \dot{\gamma} d^{2} N_{F M}^{3} \cos ^{2} \theta q_{1}(x), \Gamma_{h 2} \approx \beta \dot{\gamma} d^{2} N_{F M}^{3} \cos ^{2} \theta q_{2}(x)$

$q_{1}(x)$ and $q_{2}(x)$ being simple functions of $\mathrm{x}$, not shown here for brevity.

Equating the torque ${ }^{\Gamma_{m}}$ (eqn (3)), to the torques $\Gamma_{h 1}$ and $\Gamma_{h 2}$ (eqn (8)), we come to equations for $\theta$ for the cases $x<\sqrt{2}-1$ and $1>x>\sqrt{2}-1$ respectively. These equations include the number of FM particles in the cluster, $N_{F M}$, which can be determined by comparison of the magnetic and hydrodynamic forces acting along the cluster axis as follows. 28

a. $\mathbf{x}<\sqrt{2}-1$ and $k c=k 1$. The attractive magnetic force among the nearest particles in the cluster, can be calculated by considering the dipole-dipole approximation as: 
$F_{m 1}=\frac{\mu_{0}}{4 \pi \mu_{f}} \frac{3 m_{F M}^{2}}{d^{4}}\left(3 \cos ^{2} \theta-1\right)$

The hydrodynamic destruction force $F_{r 1}$ is estimated by summing the Stokes forces acting on the cluster particles which are below the particle of number k1 (Fig. 8b). Note that, according to the definition of $\mathrm{k} 1$, this force equals to that acting on the part of the aggregate above the point $\mathrm{k} 1$. The resulting force can be therefore calculated as:

$F_{h 1}=F_{1 x}^{\prime} \sin \theta \approx \beta \dot{\gamma} d \frac{N_{F M}^{2} \kappa_{1}^{2}(x)}{2} \sin \theta \cos \theta$

Here $\kappa_{1}$ is a simple function of $\mathrm{x}$, not shown here for brevity.

b. $\mathbf{1}>\mathbf{x}>\sqrt{\mathbf{2}}-\mathbf{1}$ and $k c=k 2$. By taking into account the interaction between (i) two nearest FM particles, (ii) two nearest DM particles and (iii) the nearest FM and DM particles, and after simple, but cumbersome calculations, we get:

$F_{m 2}=\frac{\mu_{0}}{4 \pi \mu_{f}} \frac{m_{F M}^{2}}{d^{4}}\left[3\left(1+y^{2}\right)\left(3 \cos ^{2} \theta-1\right)-\frac{y}{2^{5 / 2}}\left(12 \cos ^{2} \theta+28 \sin ^{2} \theta-9\right)\right]$

And the hydrodynamic destruction force is:

$F_{h 2}=F_{2 x}^{\prime} \sin \theta=\beta \dot{\gamma} d N_{F M}^{2}\left(1-\kappa_{2}(x)\right)^{2} \sin \theta \cos \theta$

Where $\kappa_{2}$ is a simple function of $\mathrm{x}$, not shown here for brevity.

\subsection{Macroscopic stress}

The macroscopic stress of the suspension can be estimated on the basis of the considerations made by Martin and Anderson (ref.28) as $\sigma=\eta_{0} \dot{\gamma}+\sigma_{a}$, where $\eta_{0}$ is the viscosity of the carrier and $\sigma_{a}$ is the stress produced by the aggregates, which can be expressed as ${ }^{\sigma_{a}=\frac{1}{2} n_{a} \Gamma_{m}} .28$ Here, $n_{a}=\varphi_{F M} / N_{F M} V$ is the number of aggregates per unit volume of the system, $V$ being the particle volume. By replacement with eqn (3) in the previous expression of ${ }^{\sigma}$, we get:

$\sigma_{a}=\frac{9}{8} \mu_{0} \mu_{f} \beta^{2} H_{0}^{2} \varphi_{F M} g(x) \frac{\tan \theta}{1+\tan ^{2} \theta}$

with $g(x)=1+x\left(y+y^{2}\right)$. The angle $\theta$ can be obtained as a function of $\mathrm{x}$ and $\mathrm{y}$ by solving the system of equations $\Gamma_{m}\left(\theta, N_{F M}\right)=\Gamma_{h j}\left(\theta, N_{F M}\right), F_{m j}\left(\theta, N_{m}\right)=F_{h j}\left(\theta, N_{m}\right), \mathrm{j}=1,2$ for the cases $1>x>\sqrt{2}-1$ (i.e., $\mathrm{kc}=\mathrm{k} 1)$ and $1>x>\sqrt{2}-1 \quad\left(k_{c}=k_{2}\right)$, respectively. Once the angle $\theta$ is known, we finally estimate the viscous stress $\sigma_{a}$ for the regions of relative concentrations of DM and FM particles $x<\sqrt{2}-1$ and $1>x>\sqrt{2}-1$ respectively. Such stress, in agreement with ref. 28 , does not depend on the shear rate, $\dot{\gamma}$ and, therefore, can be considered as the dynamic yield stress of the system. A dimensionless stress can be alternatively calculated as in eqn (2):

$s(x)=\frac{8 \sigma_{a}}{9 \mu_{0} \mu_{f} \beta_{F M}^{2} H_{0}^{2} \varphi_{F M}}=g(x) \frac{\tan \theta}{1+\tan ^{2} \theta}$

The theoretical dimensionless yield stress has been plotted in Fig. 5 together with the experimental data. As observed, the theory agrees rather well with the experimental results and predicts the correct order of magnitude and the trend for the dimensionless yield stress. Nevertheless, the theory underestimates the dimensionless yield stress at medium-to-high DM particle concentration. The likely reason for this underestimation is that our theory does not take into consideration the appearance of the above-mentioned exclusion areas for the 
formation of FM chains due to the presence of the DM particles. Out of these areas (permitted zones), there is an increase of the effective concentration of FM particles. This would result in such a faster-than-linear increase of the yield stress, because the dependency of the yield stress on the FM volume fraction is usually faster than linear for suspensions of FM particles.1 Because the number of exclusion areas increases with the DM volume fraction, it is logical that at medium-to-high DM particle concentration, the contribution of the exclusion areas to the experimental yield stress must be stronger. The step-wise decrease of theoretical s at $x=\sqrt{2}-1 \approx 0.41$ is explained by the transition from the situation when $\mathrm{kc}=\mathrm{k} 1$ to $\mathrm{kc}=\mathrm{k} 2$. Of course, because in the real system there will be a distribution of chains over the whole number of DM and FM particles, this transition will take place at a different place in each chain (i.e., different chains will have different kc) and, consequently, we cannot expect to experimentally observe the step-wise fall.

\section{Conclusions}

In this work we studied a new type of field-responsive fluids constituted by diamagnetic (DM) and ferromagnetic (FM) microparticles dispersed in ferrofluids, to which we referred as inverse magnetorheological (IMR) fluids. Microscopic observations in IMR fluids showed that upon magnetic field application, both the FM and the DM particles aligned in the direction of the field, giving rise to the appearance of alternating bands of particles of both populations. The formation of such structures was explained on the basis of the dipolar interaction force between particles, which was attractive when equal particles (either DM or FM) were aligned in the field direction. Nevertheless, the force between dissimilar particles was repulsive in this configuration and attractive when they were aligned perpendicularly to the field.

The field-induced rheological behaviour of IMR fluids was enhanced with respect to suspensions of FM particles containing the same amount of FM particles. What is more, we even found that the rheological behaviour was enhanced when up to $30 \%$ of the FM particle content was replaced with DM particles to obtain an IMR fluid. The enhancement of the field-induced rheological behaviour could not be explained just by the contribution of the DM chains, since the yield stress and the viscoelastic moduli for a sample with just DM particles in a ferrofluid were very low. We explained such synergistic effects by two different physical phenomena: (i) the formation of exclusion areas for FM particles as a consequence of the perturbation of the magnetic field provoked by the presence of DM particles, and (ii) the dipole-dipole interaction between DM and FM particles, which enhanced the fieldinduced particle structures.

In order to quantify the relative importance of these two phenomena, we finally developed a theoretical model which only took into account the dipole-dipole interaction among particles, but not the effect of the exclusion areas. Our model correctly predicted the order of magnitude and trend of a dimensionless yield stress. However, the theoretical model progressively underestimated the values of the yield stress as the concentration of DM particles was increased, which was supported by the fact that exclusion areas became increasingly important as the DM particle content increased.

The studied IMR fluids could be of potential interest for technological purposes, since in addition to an enhancement of the field-induced rheological behaviour, the presence of a ferrofluid as carrier reduces the sedimentation of FM microparticles, as pointed out in a previous work.13 Finally, the enhancement of the field-induced rheological response caused by the partial replacement of FM particles with DM particles would also allow producing more compact devices -i.e., smaller devices with the same power. In the long term, all these 
features, together with the lower price of the DM particles, would benefit their cost competitiveness and commercialization.

\section{Acknowledgements}

Projects 12-01-00132, 13-02-91052, 13-01-96047, 14-08-00283 (Russian Fund of Fundamental Investigations), 2.1267.2011 (Ministry of Education of Russian Federation), the Act 211 (Government of the Russian Federation № 02.A03.21.0006). The University of Granada (Acción Integrada con Rusia; Plan Propio 2011). L. Rodríguez-Arco acknowledges financial support by Secretaría de Estado de Educación, Formación Profesional y Universidades (MECD, Spain) through its FPU program.

\section{Notes and references}

a Department of Applied Physics, University of Granada, Avda. Fuentenueva s/n, 18071, Granada (Spain).

b Department of Mathematical Physics, Ural Federal University, Lenin Av. 51, 620083, Ekaterinburg (Russia).

c Faculty of Chemistry, Maria Curie-Skłodowska University, Maria Curie-Sklodowska sq. 3, 20-031 Lublin (Poland).

* Corresponding author email: modesto@ugr.es

1 A. Yethiraj, Soft Matter, 2007, 3, 1099-1115.

2 G.M. Whitesides and B. Grzybowski, Science, 2002, 295, 2418-2421.

3 M. Grzelczak, J. Vermant, E.M. Furst and L.M. Liz-Marzán, ACS Nano, 2010, 4, 35913605 .

4 L. Zhang, B.J. Nelson and L. Dong, in Encyclopedia of Nanotechnology, ed. B. Bhushan, Springer Verlag GmbH, Heidelberg, 2012, p. 1264.

5 A. Snezhko, M. Belkin, I.S. Aranson and W.K. Kwok, Phys. Rev. Lett., 2009, 102, 118103.

6 P. Tierno, T.M. Fischer, T.H. Johansen and F. Sagués, Phys. Rev. Lett., 2008, 100, 148304.

7 R.M. Erb, H.S. Son, B. Samanta, V.M. Rotello and B.B. Yellen, Nature, 2009, 457, 9991002 .

8 K.S. Khalil, A. Sagastegui, Y. Li, M.A. Tahir, J.E.S. Socolar, B.J. Wiley and B.B. Yellen, Nat. Commun., 2001, 3, 794.

9 J.E. Martin and and A. Snezhko, Rep. Prog. Phys., 2013, 76, 126601.

10 M.T. López-López, A.Y. Zubarev and G. Bossis, Soft Matter, 2010, 6, 4346-4349.

11 L. Rodríguez-Arco, M.T. López-López, G. Bossis and J.D.G. Durán, ACS Appl. Mater. Interfaces, 2013, 5, 12143-12147.

12 N. Rosenfeld, N.M. Wereley, R. Radakrishnan and T.S. Sudarshan, Int. J. Mod. Phys. B, 2002, 16, 2392-2398.

13 M.T. López-López, J. de Vicente, G. Bossis, F. González-Caballero and J.D.G. Durán, J. Mater. Res., 2005, 20, 874-881.

14 D. Susan-Resiga, D. Bica and L. Vékás, J. Magn. Magn. Mater., 2010, 322, 3166. 
15 B.J. de Gans, H. Hoekstra and J. Mellema, Faraday Discuss., 1999, 112, 209-224.

16 R. Saldívar-Guerrero, R. Richter, I. Rehberg, N. Aksel, L. Heymann and O.S. RodríguezFernández, J. Chem. Phys., 2006, 125, 084907.

17 T. Liu, X. Gong, Y. Xu and S. Xuan, Soft Matter, 2014, 10, 813.

18 M.D. Krebs, R.M. Erb, B.B. Yellen, B. Samanta, A. Bajaj, V.M. Rotello and E. Alsberg, Nano Lett., 2009, 9, 1812-1817.

19 M.T. López-López, J.D.G. Durán, A.V. Delgado and F. González-Caballero, J. Colloid. Interf. Sci., 2005, 291, 144-151.

20 G. Bossis, O. Volkova, S. Lacis and A. Meunier, Lect. Notes Phys., 2002, 594, 201-230.

21 H. Fröhlich, in Theory of Dielectrics: Dielectric Constant and Dielectric Loss, Clarendon Press, Michigan, 1958.

22 R.G. Larson, in The Structure and Rheology of Complex Fluids, ed. K.E. Gubins, Oxford University Press, New York, 1999, p. 553.

23 D. Weitz, H. Wyss and R. Larsen, GIT Lab. J., 2007, 11, 68-70.

24 P. Kuzhir, A. Gómez-Ramírez, M.T. López-López, G. Bossis, and A. Zubarev, J. NonNewton. Fluid., 2011, 166, 373-385.

25 M. Parthasarathy and D.J. Klingenberg, D.J., Rheol. Acta, 1995, 34, 417-429.

26 T.C. Halsey, J.E. Martin and D. Adolf, Phys. Rev. Lett., 1992, 68, 1519-1522.

27 M.T. López-López, L. Rodríguez-Arco, A. Zubarev, L. Iskakova, and J.D.G. Durán, J. Appl. Phys., 2010, 108, 083503.

28 J.E. Martin, and R. Anderson, J. Chem. Phys., 1996, 104, 4814-4827. 


\section{FIGURES}
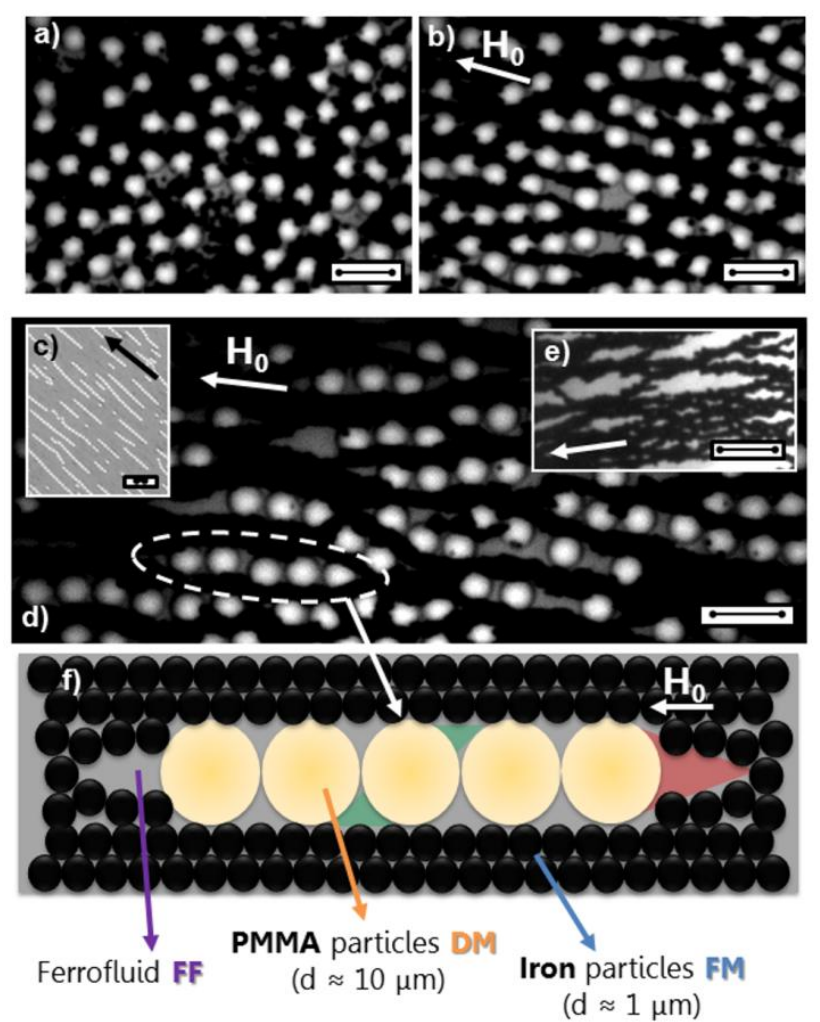

Fig. 1 (a) Initial state of randomly dispersed FM (black zones) and DM (white spheres) particles in sample FM10-DM20 at zero field. (b) Assembly of alternating bands of FM and DM chains upon the application of a magnetic field. (c) Field-induced chains of DM particles in the inverse FF, FM0-DM10. (d) A clearer picture of the field-induced chains in a different zone of sample FM10-DM20. (e) FM chains for a sample without DM particles, FM10-DM0, in which the FM chains appeared more separated. (f) Sketch of a chain of DM particles (big spheres) surrounded by dense clusters of FM particles (small spheres). Two of the pure FF zones have been highlighted: the ends of the DM chain and the interstices between DM particles. Bar lengths: 20 microns. Arrows designate the direction of the external magnetic field, $\mathrm{HO}$, of intensity $9.8 \mathrm{kA} / \mathrm{m}$. 


\begin{tabular}{|c|c|c|}
\hline $\begin{array}{l}\text { Particle } \\
\text { pair }\end{array}$ & $\begin{array}{c}\text { Space } \\
\text { configuration }\end{array}$ & $\begin{array}{c}\text { Force on } \delta \text { due to } \\
\qquad \alpha, \mathrm{F}_{\alpha \delta}\end{array}$ \\
\hline & 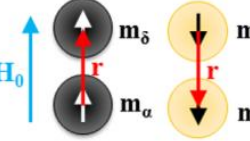 & $\mathbf{F}_{\alpha \tilde{o}}=-\frac{3 \mu_{0} m_{\alpha} m_{\delta}}{2 \pi \mu_{f} r^{5}} \mathbf{r}$ \\
\hline DM-DM & $\underset{\mathbf{m}_{\tilde{\delta}}}{\stackrel{\mathrm{r}}{\boldsymbol{r}} \underset{\mathbf{m}_{\alpha}}{\downarrow}}$ & $\mathbf{F}_{a \tilde{\delta}}=\frac{3 \mu_{0} m_{\alpha} m_{\delta}}{4 \pi \mu_{f} r^{5}} \mathbf{r}$ \\
\hline \multirow{2}{*}{ FM-DM } & $\mathrm{H}_{0} \uparrow$ & $\mathbf{F}_{\alpha \hat{\delta}}=\frac{3 \mu_{0} m_{\alpha} m_{\delta}}{2 \pi \mu_{f} r^{5}} \mathbf{r}$ \\
\hline & $\underset{\mathrm{m}_{\delta}}{\mathrm{r}} \underset{\mathrm{m}_{\alpha}}{\mathrm{T}}$ & $\mathbf{F}_{\alpha \grave{\delta}}=-\frac{3 \mu_{0} m_{\alpha} m_{\delta}}{4 \pi \mu_{f} r^{5}}$ \\
\hline
\end{tabular}

Fig. 2 Force between magnetised particles based on the dipole-dipole approximation for different space configurations.

a)

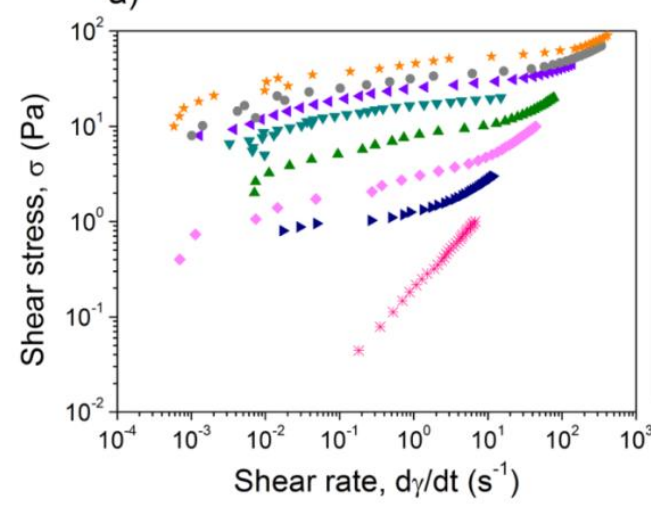

b)

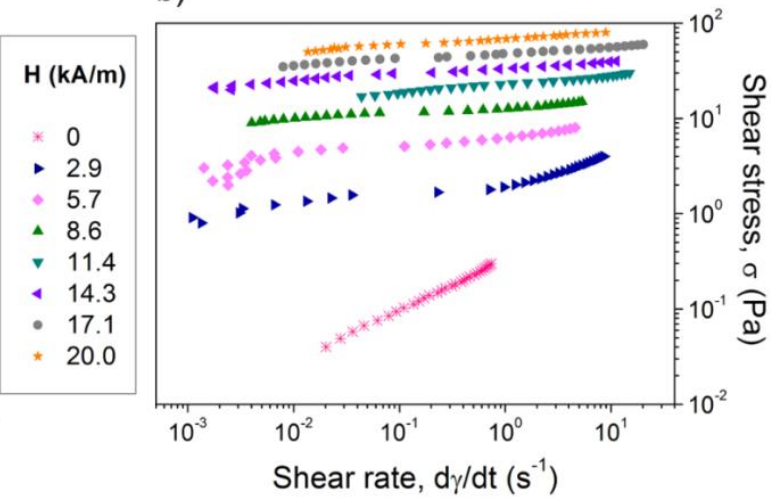

Fig. 3 Rheograms (shear stress vs. shear rate plots) of samples FM10-DM0 (a) and FM10DM10 (b). The different symbols correspond to different values of the external magnetic field, H0. The rheological behavior in the absence of field was Newtonian for both samples and changed to a plastic behavior when the field was activated. The shear stress at a given shear rate was usually higher in the case of sample FM10-DM10 for all the applied fields. 

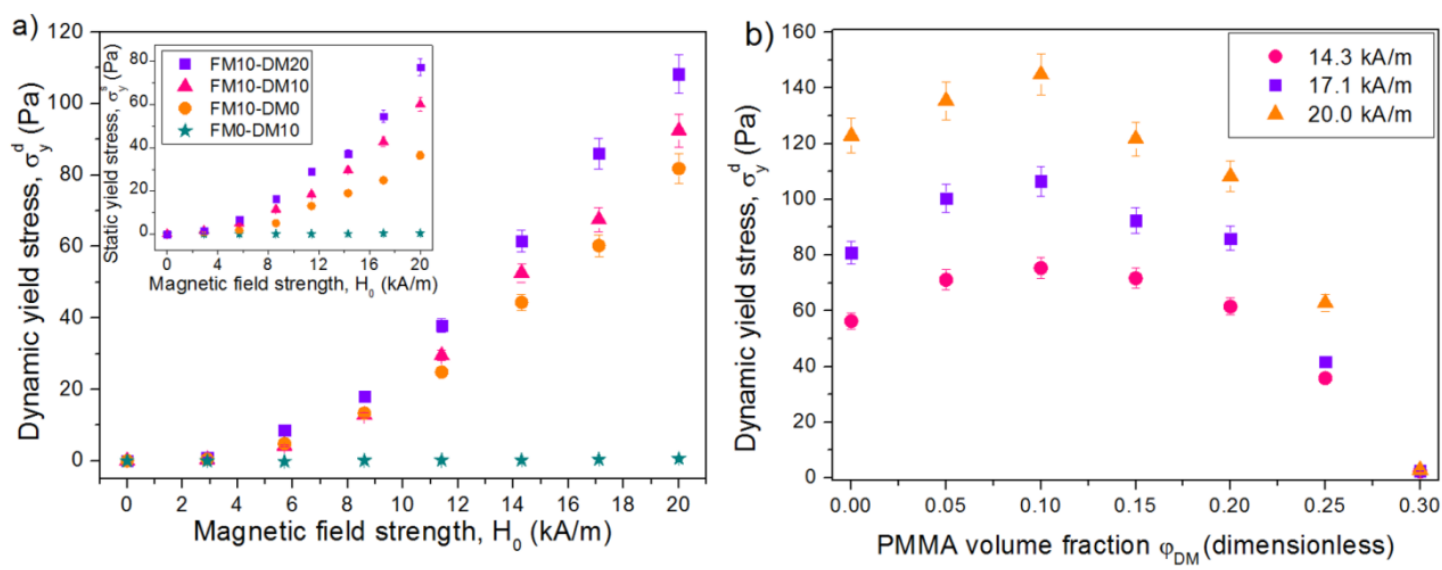

Fig. 4 (a) Dynamic yield stress as a function of the applied magnetic field for all the samples:

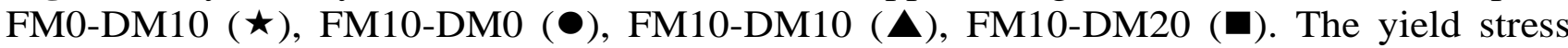
was enhanced when DM and FM particles were mixed together, increasing with the DM volume fraction. The yield stresses of samples FM10-DM0 and FM0-DM10 were lower for all the applied fields. In the latter case it was almost negligible in comparison. Similar results were obtained for the static yield stress (inset), although in this second case, its relative increase with the DM volume fraction was higher. (b) Dynamic yield stress as a function of the DM volume fraction, $\varphi_{D M}$, for different intensities of the applied magnetic field and constant total volume fraction $\varphi_{D M}+\varphi_{F M}=0.3$. The yield stress exhibited a maximum for a critical volume fraction of DM particles, $\varphi_{D M} \approx 0.1$. Such a maximum indicates that it was possible to increase a magnetic-related property, just by replacing $10 \%$ of FM particles with DM particles.

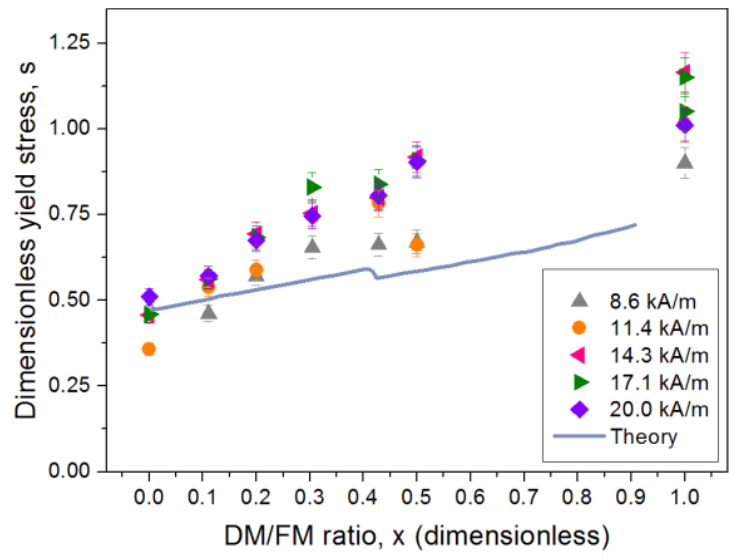

Fig. 5 Dimensionless dynamic yield stress, s, (eq. (2)) as a function of the ratio of the volume fractions of DM and FM particles, $x$. The different symbols correspond to experimental data obtained at different magnetic fields. As observed, all the experimental data followed a master curve which showed an increasing trend with $\mathrm{x}$. The line corresponds to the theoretical predictions of eq. (14), of the same order of magnitude as the experimental data. 
a)

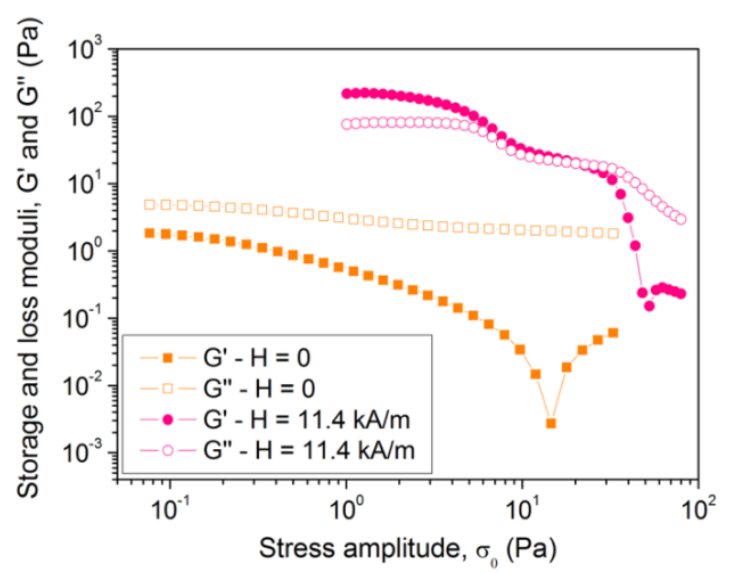

b)

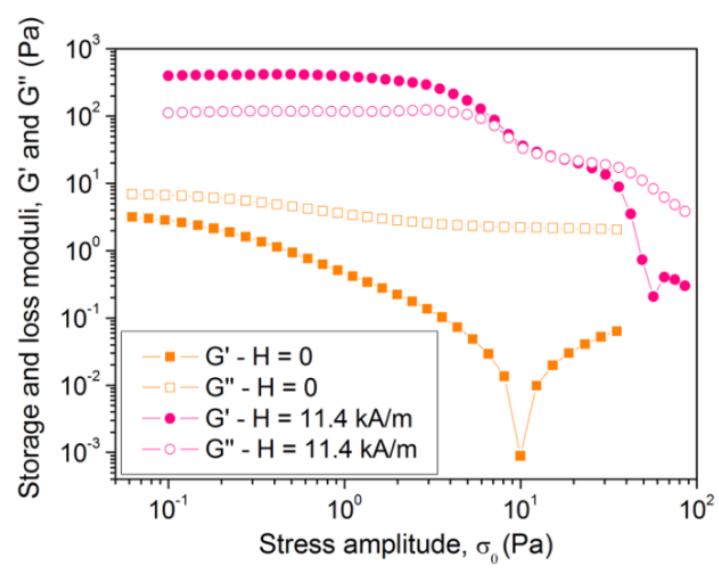

Fig. 6 Viscoelastic moduli as a function of the amplitude of the applied oscillatory stress for samples FM10-DM0 (a) and FM10-DM10 (b) at two values of the external magnetic field: 0 (squares) and 11.4 (circles) $\mathrm{kA} / \mathrm{m}$. G' and G' are represented by full and open symbols respectively. In the absence of magnetic field $G$ ' ' was always higher than $G$ '. However this behaviour reversed when the field was applied for the samples with FM particles in their composition. The values of both moduli in the first plateau were higher for the IMR fluid.
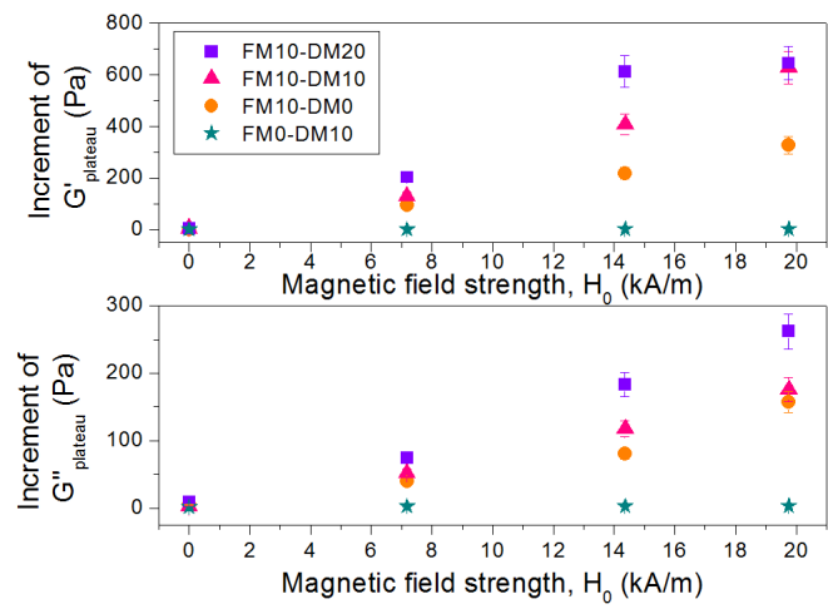

Fig. 7 Increment (with respect to their value at zero field) of the viscoelastic moduli, G' and G', at the initial plateau as a function of the applied magnetic field for all the samples: FM0-

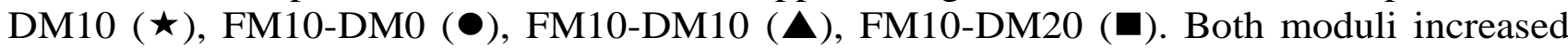
when DM and FM particles were mixed together, increasing with the DM volume fraction. G' and G', were always lower for samples FM10-DM0 and FM0-DM10. In the latter case they were almost negligible in comparison. 

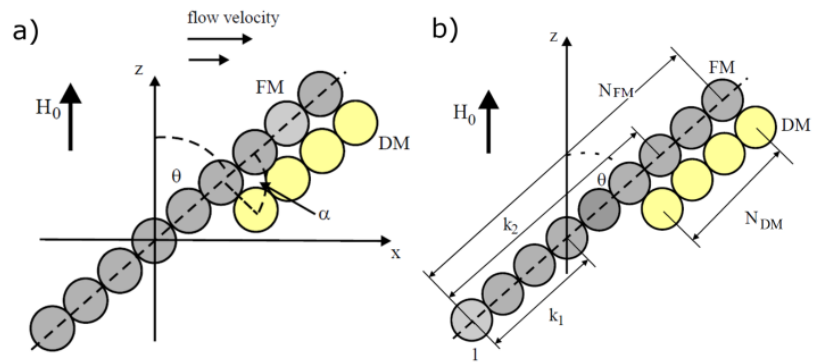

Fig. 8 (a) Sketch of the composite aggregate of DM and FM particles. For simplification, all the particles are considered to have the same size. Because the magnetic moments $\mathrm{mFM}$ and $\mathrm{mDM}$ have opposite directions, the FM and DM chains experience lateral attraction and aggregation. The extreme particles of the chains are in contact because the energy of interaction between the FM and DM particles is maximal near the extremities of the chains. (b) Same as (a) but specifying the positions of the particles. $\mathrm{k} 1$ and $\mathrm{k} 2$ are particle numbers where the condition $\dot{\gamma} z_{0}=v_{0}$ is held 